\title{
Katalaseaktivitätsbestimmung im Blut
}

\author{
Von \\ E. WERnER und H. HeIDER \\ Aus der Kinderklinik der Freien Universität Berlin (Kaiserin Auguste Victoria Haus) \\ (Direktor: Prof. Dr. A. Loescbke)
}

Der Schriftleitung zugegangen am 8. Dezember 1962

\begin{abstract}
Da die gebräuchlichen Methoden zur Katalaseaktivitätsmessung im Blut nicht voll befriedigen, wird in Anlehnung an bekannte Methoden eine Bestimmung der Katalaseaktivität nach dem Prinzip des optischen Tests beschrieben. Hinsichtlich ihrer Handhabung und geringen Fehlerbreite scheint diese Methode älteren Verfahren überlegen zu sein. Bei Untersuchungen zur Bedeutung der Katalase für den Hämoglobin- und Erythrozytenstoffwechsel wird die Berechnung eines Katalase-Hämoglobin-Index empfohlen.

Usual methods for the determination of blood catalase are not entirely satisfactory. A spectrophotometric determination, based on known methods, is described; in its operation and small error, it appears superior to older methods. Calculation of a catalase-haemoglobin index is recommended for studying the significance of catalase in haemoglobin and erythrocyte metabolism.
\end{abstract}

Für vergleichende, klinische Bestimmungen der Katalaseaktivität des Blutes muß die Methodik exakt und schnell durchführbar sein. Die Titrationsmethoden $(1,2)$ und auch die Polarographie (3) werden diesen Anforderungen nur teilweise gerecht. In Anlehnung an die von BERGMEYER für präparative Zwecke nach dem Prinzip des optischen Tests nach WARBurg angegebene Methode (4) und unter Berücksichtigung der Versuchsbedingungen älterer Methoden (1, 3, 4, 5) haben wir die Katalaseaktivität des Blutes spektrophotometrisch bestimmt.

Zur Bestimmung eignet sich insbesondere die Fähigkeit der Katalase, Wasserstoffperoxyd zu spalten. Verfolgt man die Abnahme einer bestimmten $\mathrm{H}_{2} \mathrm{O}_{2}$-Konzentration unter der Einwirkung der Katalase und trägt die nacheinander. ermittelten Restmengen des Wasserstoffperoxyds gegen die Zeit auf, so ergibt sich eine Kurve, deren Verlauf einer monomolekularen Reaktion entspricht (6). Für die $\mathrm{H}_{2} \mathrm{O}_{2}$-Zerlegung durch die Katalase gilt aber nur unter bestimmten Bedingungen die Reaktionskonstante der monomolekularen Reaktion, wie im folgenden dargelegt wird:

Die Reaktionskonstante $K$ wird um so eher bestätigt, je niedriger die Wasserstoffperoxydkonzentration ist. $\mathrm{Da}$ das Substrat in größeren Konzentrationen die Katalase hemmt, müssen $\mathrm{H}_{2} \mathrm{O}_{2}$ Konzentrationen von $0,005-0,015 m$ gewählt werden $(7,8)$.

Das pH-Optimum der Katalasereaktion liegt bei $\mathrm{pH}=7,0(7,8,9)$. Eine Inaktivierung der Katalase erfolgt oberhalb $\mathrm{pH}=10,3-11,0$ und unterhalb $\mathrm{pH}=\mathbf{5 , 0}$. Temperaturerhöhungen können zu Aktivitätsverlusten führen. Die Temperaturempfindlichkeit der Katalase steigt mit der Verdünnung und der Gegenwart von Wasserstoffperoxyd (9). Das Temperaturoptimum der Katalase liegt bei $+2^{\circ}(7,8)$. Einen Aktivitätsverlust können auch mechanische Einwirkungen (Schütteln, Filtrieren usw.) und Strahlen herbeiführen. Die UV-Strahlung hemmt besonders bei optimalem $\mathrm{pH}$ die Katalase in verdünnten Lösungen (9). Die Katalase wird durch alle Stoffe, die den Kolloidzustand des Fermentes beeinflussen, geschädigt (8). Über zahlreiche Hemmstoffe berichtet die Literatur $(10,11,12)$. Nach Brschopf ist der Katalaseindex des Blutes aus dem Ohrläppchen böher als aus dem Finger oder der
Ferse (13). - Die Versuchsdaucr sollte vor allem bei ungereinigten Katalasepräparaten möglichst kurz sein. Im Verlauf der Reaktion nimmt die Aktivität der Katalase um so mehr ab, je größer der Verdünnungsgrad des Fermentes zu Beginn der Untersuchung ist (4). Mitverantwortlich für den Aktivitätsverlust der Katalase mag sein, da $\beta$ die Temperaturinaktivierung in Gegenwart von Wasserstoffperoxyd bei den vorwiegend bei Zimmertemperatur ablaufenden Versuchen schon sehr deutlich wird. Bei Blutkatalasepräparaten, die durch Hämolyse gewonnen werden, gehen gleichzeitg Stoffe in Lösung, die zunehmend eine Katalaseinaktivierung bewirken. Dafür werden u. a. Proteasen und eine Antikatalase mit wechselnder Aktivität verantwortlich gemacht $(9,1)$.

\section{Bestimmung der Katalaseaktivität nach dem Prinzip des optischen Tests nach WARBURG.}

Spektrophotometrisch ist eine objektive Bestimmung der Substratkonzentration zu jedem Zeitpunkt der katalatischen $\mathrm{H}_{2} \mathrm{O}_{2}$-Spaltung möglich, $\mathrm{da}$ Wasserstoffperoxyd deutliche Absorption im UV-Bereich zeigt (14). Die Anfangsextinktion $E_{0}$ der $\mathrm{H}_{2} \mathrm{O}_{2}$-Lösung mit der Konzentration $c_{0}$ erreicht nach Zugabe einer bestimmten Katalasemenge im Zeitpunkt $t$ den Wert $E_{t}$ entsprechend der $\mathrm{H}_{2} \mathrm{O}_{2}$-Konzentration $c_{l}$. Die für die katalatische $\mathrm{H}_{2} \mathrm{O}_{2}$-Zersetzung geltende Gleichung einer monomolekularen Reaktion:

$$
K=\frac{1}{t} \cdot \log \frac{c_{0}}{c_{t}}
$$

läßt sich nach den gefundenen Extinktionswerten wie folgt umformen:

$$
K=\frac{1}{t} \cdot \log \frac{E_{0}}{E_{t}}
$$

\section{Metbodik}

Die Messungen werden mit dem Spektralphotometer DU der Firma Beckman bei $\lambda=240 \mathrm{~m} \mu$ durchgeführt. Die benutzten Glasgeräte sind geeicht. Als Reagenzien dienen $\mathrm{H}_{2} \mathrm{O}_{2}$ („Perhydrol"-Merck) und 
Phosphatpuffer $\mathrm{pH}=7,0 \quad(0,05 \mathrm{~m})$. Das Blut wird mittels Hb-Pipette aus der Fingerkuppe entnommen. Die Konzentration des Testsubstrates wird täglich neu eingestellt. Dazu werden $0,08 \mathrm{~m} l$ Perhydrol-Merck mit $0,05 \mathrm{~m}$ Phosphatpuffer $\mathrm{pH}=7,0$ auf $50 \mathrm{ml}$ aufgefüllt. Diese Lösung gemessen gegen $0,05 \mathrm{~m}$ Phosphatpuffer bei $240 \mathrm{~m} \mu$ ergibt eine Extinktion von etwa 0,500 . Es wird mit Phosphatpuffer weiter verdünnt, bis die Extinktion $E_{0}=0,400 \pm 0,020$ entsprechend einer $0,009 \mathrm{~m} \mathrm{H}_{2} \mathrm{O}_{2}$-Lösung beträgt. Dieses. $\mathrm{H}_{2} \mathrm{O}_{2}$-Puffergemisch wird bei $20^{\circ}$ im Wasserbad inkubiert. - Das Katalasepräparat wird gewonnen, indem $20 \mu l$ Blut mit einer Hb-Pipette entnommen und in $25 \mathrm{ml}$ eisgekühltem aqua bidest. sofort hämolysiert werden. Das Hämolysat wird im Eisbad aufbewahrt und innerhalb $30 \mathrm{Min}$. nach der Entnahme verarbeitet. Für die Reaktion werden $3 \mathrm{~m} l \mathrm{H}_{2} \mathrm{O}_{2}$-Puffergemisch in eine Silica-Quarz-Küvette pipettiert. Es wird die Extinktion $E_{0}$ gegen $0,05 \mathrm{~m}$ Phosphatpuffer bestimmt. Danach mischt man mittels Glasstab schnell $0,2 \mathrm{ml}$ Hämolysat ein und stoppt die Zeit. Die folgenden Extinktionswerte $E_{t}$ werden nach $1,2,3$ und 4 Min. abgelesen. Eine Kühlvorrichtung hält die Temperatur im Photometer bei $20^{\circ} \pm 0,5^{\circ}$.

Ausvertung: Die Extinktionswerte $E_{0}$ und $E_{t}$ werden in die Formel (2) für die Geschwindigkeitskonstante $K$ der monomolekularen Reaktion eingesetzt und die $K_{t}$-Werte für die 1., 2., 3. und 4. Minute errechnet. Das arithmetische Mittel ergibt den $K$-Wert für das gemessene Katalasepräparat.

\section{Der Katalase-Index}

Die Katalase ist im Serum, in den Leukozyten, Thrombozyten und Erythrozyten vertreten (9). Die größte Menge der Blutkatalase entfällt jedoch auf die Erythrozyten (15). Für Aktivitätsvergleiche ist ein Katalase-Erythrozyten-Index (16) eingeführt worden, da die Aktivität. weniger vom verwendeten Blutvolumen, als in erster Linie von der Erythrozytenzahl abhängig ist. Dieser Katalase-Erythrozyten-Index ist der Quotient aus der mit verschiedenen Methoden gemessenen Katalaseaktivität $K$ eines Hämolysates und der Zahl der Erythrozyten in Millionen pro $\mathrm{mm}^{3}$ Blut. In Anlehnung an JoNes und McCANCE (17), die die Katalaseaktivität pro mg Bluteisen ausdrücken, bezieht ScHürz (3) die Katalaseaktivität des Blutes auf den Hämoglobingehalt. $\mathrm{Da}$ die Rolle der Katalase für die Erhaltung des Hämoglobins diskutiert wird, interessiert besonders das Verhältnis der Blutkatalaseaktivität zum Hämoglobin. Es wird deshalb bei allen eigenen Untersuchungen ein Katalase-Hämoglobin-Index berechnet (10), indem die Reaktionskonstante $K$ der katalatischen $\mathrm{H}_{2} \mathrm{O}_{2}$-Spaltung durch den Hämoglobingehalt in $\mathrm{g} \%$ der gleichen Blutprobe dividiert und zur Vereinfachung der Schreibweise mit 100000 multipliziert wird:

$$
\mathrm{KI}_{\mathrm{Hb}}=\frac{K}{\mathrm{~g}_{0} / \mathrm{Hb}} \cdot 100000 .
$$

Das Hämoglobin wird photometrisch als Cyanhämiglobin mit einer Genauigkeit von $\pm 1 \%$ bestimmt (9).

\section{Diskussion}

Unter den angegebenen Versuchsbedingungen liefert die $\mathrm{H}_{2} \mathrm{O}_{2}$-Spaltung durch die Katalase des Blutes weitgehend konstante $K$-Werte. Geringe Abweichungen gehen zurück auf den unvermeidlichen Aktivitätsverlust der Blutkatalase durch die Anwesenheit von Antikatalase und Proteasen, durch die UV-Strahlung und die Temperatur von $20^{\circ}$ bei der Messung im Beckman-Photometer. Die Reproduzierbarkeit so gewonnener Werte eines bestimmten Individuums an verschiedenen Tagen ist mit einem relativen Fehler von $\pm 1,4 \%$ behaftet. Weicht die Temperatur bei $20^{\circ}$ um $0,5^{\circ} \mathrm{ab}$, so ändert sich die relative Aktivität der Katalase um 2,6\% (18). Die Titrationsmethode bestimmt das testliche $\mathrm{H}_{2} \mathrm{O}_{2}$ zu einem festgelegten Zeitpunkt seiner katalatischen Spaltung mittels Jodometrie oder Manganometrie. - Für die Jodometrie wird eine Fehlerbreite von $\pm 10 \%$ angegeben (2). Infolge ihrer relativ langsamen Reaktion können Fehler durch Luftoxydation des Jodwasserstoffes entstehen. Ferner wird nicht nur durch Wasserstoffperoxyd, sondern auch durch die Katalase selbst 'Jod freigesetzt (8). Das Jod schädigt wiederum die Katalase (9). Durch die Titration mit $\mathrm{Na}_{2} \mathrm{~S}_{2} \mathrm{O}_{3}$ erfaßt man auch die Sauerstoffmenge, die durch die $\mathrm{H}_{2} \mathrm{O}_{2}$-Zerlegung entwickelt wird. - Die Titration des $\mathrm{H}_{2} \mathrm{O}_{2}$ mit $\mathrm{KMnO}_{4}$ kann durch Reduktion des Permanganates in ungereinigten Katalaselösungen gestört werden (8). Aưch die Beurteilung des Farbumschlages nach „zartrosa“ in sehr verdünnten Lösungen ist subjektiven Schwankungen unterworfen. Ferner vergrößert das relativ schnelle Absinken des Titers bei länger dauernden Reihenuntersuchungen die Fehlerbreite (3). - Die Polarographie nach ScHüTz (3) ermittelt die nach der Katalasereaktion verbleibende Restmenge Wasserstoffperoxyd und den freigesetzten Sauerstoff, die proportionale Stufenhöhen im Polarogramm ergeben. Es wird eine Schwankungsbreite der Ergebnisse von $\pm 7,7 \%$ beschrieben. Ungenauigkeiten können durch den zur polarographischen Untersuchung nötigen Tylosezusatz entstehen. Er verhindert eine schnelle und gleichmäßige Verteilung des Reaktionsgemisches in der Grundlösung des Polarographen, wodurch falsche Stufenhöhen im Polarogramm entstehen. Außerdem erstreckt sich die Versuchsdauer auf 30 Minuten, in der bereits eine merkliche Katalaseinaktivierung einsetzt. Der $K$-Wert wird durch Extrapolation nach $t_{0}$ gewonnen, was eine zusätzliche Fehler'quelle bedeutet. Wenn Scrürtz eine $0,018 \mathrm{~m} \mathrm{H}_{2} \mathrm{O}_{2}$ Lösung und eine Blutverdünnung von 1:25000 wählt, liegen bei den eigenen Untersuchungen insofern günstigere Konzentrationsverhältnisse $\left(0,009 \mathrm{~m} \mathrm{H}_{2} \mathrm{O}_{2}\right.$ Lösung, Blutverdünnung 1:19000) vor, als die Stabilität der Katalase um so größer wird, je geringer ihr V.erdünnungsgrad $(4,19)$ und die $\mathrm{H}_{2} \mathrm{O}_{2}$-Konzentration $(7,8)$ zu Beginn des Tests sind. - Die photometrische Messung der Katalaseaktivität mit „fliegendem Start" 
nach BergmeYer (4) zeigt eine Fehlerbreite von $\pm 2 \%$. Die Ergebnisse resultieren aus einer Ablesung während der Katalasereaktion. Ablesungsfehler lassen sich korrigieren, wenn man mehrere Messungen in einem kurzen Reaktionsabschnitt vornimmt und zu einem Durch- schnittswert zusammenfaßt (10). - Die Vorteile der hier beschriebenen Methode zur Katalaseaktivitätsbestimmung für klinische Zwecke bestehen im Vergleich zu älteren Methoden in ihrer schnelleren Durchführung und größeren Genauigkeit.

\title{
Literatur
}

1. HelleR, H., Röntgen-Laborat. praxis, Stuttgart 10, 183 (1957). - 2. Lück, H., Biochem. Z. 328, 411 (1957). - 3. ScHürz, E., Übet die Katalaseaktivität des Säuglingsblutes und ihre Bestimmung mittels Polarographie, Med. Diss., Würzburg (1954). 4. BergmeYer, H. U., Biochem. Z. 327, 255 (1955). - 5. Herbert, D., Methods in Enzymology Vol. II, 764, 780 (1955). - 6. SENTER, G., Z. physik. Chem. 44, 257, (1903). - 7. v. EuLER, H. und K. Josephson, Liebigs Ann. Chem. 452, 158 (1927); 455, 1 (1927). -8. Zerle, K., Katalase, S. 2615 aus Bamann, E. und K. Myrbäck, Die Methoden der Fermentforschung III, Georg Thieme, Leipzig (1941). - 9. v. Euler, H. und K. ZmILE, Die Katalasen, aus v. Euler, H., Chemie der Enzyme II, 3. Bergmann, München S. 663 (1934). - 10. Herder, H., Die Blutkatalaseaktivität im
Kindesalter und ihre Beeinflussung durch bestimmte Erkrankungen und Vitamin K-Präparate, Med. Diss., Berlin (1962). - 11. Hoffmann-Ostenhof, O., Fortschr. Chem. org. Naturstoffe (Wien) 6, 154 (1950). - 12. SErde, G., Biochem. Z. 308, 175 (1941). -13. Brschoff, H., Arch. Kinderhk., Stuttgart 82, 189 (1927). 14. BeERS, R. F. und J. W. Sizer, J. biol. Chemistry 195, 133 (1952). - 15. Kurokawa, H., Tohoku J. Exper. Med. 14, 520, 539 (1929/30). - 16. v. ThIemen, G. J., Dtsch. Arch. klin. Med. 131, 113 (1920). - 17. Jones, P. E. H. und R. A. MCCANCE, Biochem. J. 45, 464 (1949). - 18. Morgulis, S. und M. Beber, J. biol. Chemistry 72, 91 (1926). - 19. TheoreLl, H., Experientia, Basel 4, 100 (1948).
Priv.-Doz. Dr. med. Egon Werner Kinderklinik der Freien Universität Berlin (Kaiserin Auguste Victoria Haus) 1 Berlin 19, Heubnerweg 6

\section{Isolierung und Feststellung der chemischen Zusammensetzung eines Perchlor- säure-löslichen Glykoproteids aus dem Urin von Plasmocytom-Kranken*)}

\author{
Von \\ H. WeICKer, K. HuhNSTOCK und D. GRÄSSLIN \\ Aus der Medizinischen Universitäts-Poliklinik Heidelberg (Direktor: Professor Dr. H. Plügge) \\ Meinem verehrten Lehrer, Herrn Professor S. J. Thannhauser, zum Gedächtnis
}

(Der Schriftleitung zugegangen am 6. März 1963)

\begin{abstract}
Aus dem Gesamturoprotein-Komplex von Plasmocytom-Patienten wurde durch Hydrolyse und nachfolgende Lipid-Extraktion der gefriertrocknen Substanz ein Glykopeptid isoliert, das ein Molekulargewicht von 19900 besitzt. In Ultrazentrifuge und Elektrophorese ist es homogen. Es ist in Wasser hitzestabil, in $0,6 \mathrm{~m}$ Perchlorsäure gut löslich, in 10 proz. Trichloressigșäure hịngegen unlöslich. Es enthält 16 verschiedene Aminosäuren und ist methioninfrei. Der Gesamtkohlenhydratgehalt beträgt 38,1\%. Die Kohlenhydrate haben folgende molare Verteilung: Ga:Ma:Glu:Fu:Rh:GlA:GaA:NS = 10:5:5:4:2:8:2:5. Es unterscheidet sich physiko-chemisch von anderen Perchlorsäure-löslichen Proteiden des Serums und Urins. Es dürfte für die Uroproteine des Plasmocytoms charakteristisch sein, da es im Harn von Normalpersonen und bei Proteinurien anderer Genese nicht isoliert werden konnte.
\end{abstract}

A glycopeptide, molecular weight 19.900, was isolated from the total uroprotein complex of plasmacytoma patients by lipid extraction of the freeze-dried product after hydrolysis. It is homogeneous in the ultracentrifuge and in electrophoresis. It is thermostable in water, very soluble in $0.6 \mathrm{M}$ perchloric acid and insoluble in $10 \% \mathrm{TCA}$. It contains 16 different amino acids and no methionine. The total carbohydrate is $38.1 \%$ and of the following molar composition: $\mathrm{Ga}: \mathrm{Ma}: \mathrm{Glu}: \mathrm{Fu}: \mathrm{Rh}: \mathrm{GlA}: \mathrm{GaA}: \mathrm{NA}=10: 5: 5: 4: 2: 8: 2: 5$. Physicochemically, it is different from other perchloric acid-soluble proteins of serum and urine. It could not be found in normal urine or that from other types of proteinuria; it may thus be a characteristic uroprotein of plasmacytoma.

*) Mit Unterstützung der Deutschen Forschungsgemeinschaft. 